\title{
Le Dipoula de Sti et Pahé : un Titeuf mal noirci ? Endroit et envers de la Francophonie à travers une bande dessinée franco- gabonaise
}

\author{
Ludovic OBIANG \\ Institut de Recherche en Sciences Humaines \\ (CENAREST GABON)
}

\section{Introduction}

S'il est un projet qui reste controversé dans son principe et ses motivations, c'est bien celui de la Francophonie, tel qu'il a été établi conjointement par l'ancienne puissance coloniale française et par certains représentants prestigieux de ses anciennes colonies. L'essentiel de la défiance porte sur une forme de domination insidieuse, aussi bien linguistique que politique, les critiques les plus virulents allant jusqu'à dénoncer une tentative de "camoufler l'entreprise néocoloniale, pervertissante et profondément mensongère des puissances financières de l'ancienne métropole sur les fragiles économies du continent» (Ziegler Main 9). Malgré les multiples recadrages et plaidoyers en faveur d'une communauté fondamentalement linguistique, placée sous le signe du partage et de la tolérance, le sentiment demeure celui d'une France (métonymie d'un Occident) toute puissante, qui satellise une multitude de nations échelonnées selon leurs potentialités économiques. Ce sentiment est renforcé par le constat d'une prépondérance de la partie occidentale chaque fois qu'il est question de concevoir et de mettre en œuvre un projet à caractère francophone, les institutions occidentales, fortes de leur poids financier, optant pour une logique d'universalisme tous azimuts, qui fait peu cas de la spécificité du plus petit. Un exemple, parmi tant d'autres, de ces projets hybrides et déséquilibrés, est fourni par la bande dessinée Dipoula l'albinos (Mbolo), née de la collaboration entre trois parties, un éditeur et un scénariste français (Paquet et Sti) et un dessinateur gabonais (Pahé).

Le texte affirme pêle-mêle différentes intentions louables, en particulier celle de dénoncer la stigmatisation de l'albinos et le rejet de l'orphelin dans le cadre de la société gabonaise. Projet méritoire, à condition que le scénariste ne se contente pas d'une seule semaine d'immersion dans la société indexée! À moins que les traits sous lesquels il brode son Dipoula ne soient une distorsion d'un personnage de son univers familier, le célèbre Titeuf de Zep. L'on finirait ainsi par s'interroger sur le lectorat visé et sur les motivations réelles des partenaires ? Mais plus largement, c'est bien la Francophonie institutionnelle qui est ici en question : cette production n'est-elle pas la preuve qu'un rapport déséquilibré entre les forces en présence nuira toujours à l’idéal de partage et de dialogue cher à la Communauté ?

Nous développerons cette réflexion en respectant un plan en trois parties. La première articulation présentera ce que nous avons convenu d'appeler « l'endroit » du projet, c'est-à-dire le principe, noble dans sa formulation, qui consiste à faire la promotion d'un jeune talent gabonais en lui adjoignant un scénariste français émergent et en mettant leur association sous le signe d'un engagement généreux contre la stigmatisation de l'albinisme. La deuxième partie rétablira les réalités anthropologiques ou sociologiques en fonction desquels s'effectuent la caricature (stigmatisation des albinos et politique d'adoption au Gabon) de manière à fournir au lecteur les moyens d'évaluer le degré de travestissement opéré par les auteurs sur des situations qui sont autrement plus complexes. La troisième articulation examinera l'album dans une perspective essentiellement descriptive, afin de mettre au jour «l'envers» de l'entreprise, à savoir les mécanismes inavoués d'une européanisation de Dipoula (ou d'une africanisation de Titeuf) 
dévolue à des intérêts commerciaux et susceptible de véhiculer une dévalorisation insidieuse de l'Africain.

\section{1) Le projet Dipoula : de «l'inécolier» rebelle (1985) à l'albinos orphelin (2008)}

S'il est une croyance à laquelle Pahé, de son vrai nom Patrick Essono, s'accroche, c'est d'attribuer à la bonne fortune et au hasard les fruits d'un talent qui n'en est pas moins remarquable. Ainsi déclarera-t-il à Nicolas Vadeau du site Bulle d'encre: «Un vrai coup de chance que la vie de Moi » (Vadeau Rencontre). Propos qu'il confirmera un an plus tard auprès d'Armel Le Ny, un journaliste de la Charente Libre: «Au Gabon, il y a une petite fée qui est venue me taper sur la tête avec sa baguette magique » (Le Ny Vacances). Et l'on peut admettre effectivement qu'il est né coiffé, à en croire les grandes étapes qui articulent sa carrière. Toujours à Armel Le Ny :

C'est vrai que j'ai une vie chanceuse. En 2003, je publie dans un collectif au Cameroun. En 2004, un éditeur fou tombe dessus. En 2005, il me fait signer un contrat. En 2006 sort mon premier album, «La vie de Pahé», qui raconte mon enfance. En 2007, au festival d'Angoulême, une productrice achète les droits pour en faire un dessin animé. Si c'est pas de la chance, ça! (idem)

Cette «chance» aura été particulièrement à l'œuvre dans le cas de Dipoula dont les aventures commencent en 1985, alors que Pahé est encore au lycée, à Libreville. S'en ouvrant à Nicolas Vadeau, il lui déclare : "Dipoula est un projet qui date de 1985 lorsque j'étais encore sur les bancs du lycée, en 5e au lycée d'Etat de l'Estuaire à Libreville. Au départ, Dipoula n'était pas encore albinos mais "pompeur et expert en tricherie". "Dipoula le pompeur" apparait donc dans les pages du journal L'ECLAIR du lycée ». (Vadeau Rencontre)

Dipoula est donc à l'origine un lycéen frondeur de 10 à 12 ans, qui se singularise par son habilité à transgresser les interdits scolaires. "Albinos", il ne l'est encore que symboliquement, dans la mesure où Pahé lui transmet des frustrations qui sont d'abord les siennes, celles de ne pas trouver sa vraie place, étranger dans cette France d'accueil qu'il vient de quitter et exilé dans ce Gabon natal qui le rejette.

Ce qui va changer pour Dipoula (et pour son "papa ») et le faire basculer dans l'albinisme à part entière, c'est l'intervention d'un éditeur «fou» (Le Ny Vacances) qui veut ajouter un graffiti gabonais à une collection éditoriale déjà bien fournie. À condition toutefois que Pahé veuille bien sacrifier à quelques concessions, comme perdre beaucoup de sa spécificité gabonaise pour s'ouvrir à un public plus large, européen en l'occurence. Représenté à l'époque comme un gabonais trop "francisé», Dipoula devient par la magie du crayon (ou de la gomme) un garçonnet foncièrement «couleur locale» qui ne demande qu'à s'exporter. Ecoutons Pahé s'en confier à Habib Bangré d'Afrik.com :

Le Dipoula qui paraissait dans les années $80[\ldots]$ n’avait pas le même âge et il était plus longiligne. Il portait un pantalon, une paire de baskets mais avait déjà son petit gilet. Dipoula est devenu albinos plus tard et j’ai dû effectuer plusieurs retouches. Je préfère le Dipoula actuel : il fait plus petit africain, enfant de la rue. Le premier était trop européanisé. Au départ, les scénarios étaient plus axés pour (sic) un public gabonais. En rencontrant mon éditeur, on a décidé d'internationaliser Dipoula afin qu'il puisse être accessible à tous. (Bangré Dipoula)

Cette « internationalisation» de Dipoula va s'opérer par un durcissement de la perspective, en transformant les difficultés d'adaptation du premier Dipoula en un véritable drame de stigmatisation, celui que vivent les albinos dans plusieurs pays d'Afrique, au Gabon en particulier. Pour les besoins du scénario, Pahé accepte le renfort d'un spécialiste, le Lillois Sti, de son vrai nom Ronan Lefebvre. 
De par leurs efforts conjugués, Dipoula, se transforme d'une sorte "d'inécolier » frondeur, exprimant son désarroi par un boycottage systématique des règles scolaires, en un orphelin pétulant, s'efforçant de prendre ses handicaps avec bonne humeur. Abandonné dès sa naissance par ses parents, il lui faudra l'accueil d'un orphelinat tenu par des religieuses et l'affection d'une bande de «chenapans » comme lui pour épanouir sa simple joie d'exister et son droit de vivre. La «mayonnaise» prend donc, sous la forme d'une œuvre engagée, une contribution à la réhabilitation des albinos qui, à l'instar de nombreuses bandes dessinées africaines, prendra le détour de l'humour pour « dédramatiser une histoire trop grave, parce que trop réaliste, traitant de problèmes de la vie quotidienne» (Couturier 19).

«La rigolade» est si contagieuse que l'œuvre intègre le Gang des talents, une sélection de jeunes talents parrainée par Luc Besson et la Caisse d’Épargne. Partout où elle est présentée, affirme Pahé à Habib Bangré, l'œuvre est «bien accueillie par les parents et leurs enfants » (Bangré Dipoula), au point d'être primée à maintes reprises ${ }^{1}$.

Toutefois, quelle que soit la foi de Pahé en sa "chance», elle ne peut nous faire oublier que les éditeurs se doublent de redoutables commerçants qui laissent rarement primer leur passion sur les impératifs de rentabilité. Or, les mots que Pahé prête à son éditeur traduisent bien la volonté qu’à ce dernier de sortir de son "ghetto » le personnage initial et de l'ouvrir à un public plus large, équivalent d'un marché plus étendu. Dès lors, une question s'impose : le projet «Dipoula » est-il aussi sincère qu'il se présente ? La situation dénoncée par Pahé correspond-elle à une réalité aussi localisée qu'il le prétend? En d'autres termes, Dipoula est-il aussi gabonais que Pahé et Sti veulent le faire croire?

\section{2) Dipoula un vrai-faux gabonais?}

S’il est vrai que tout œuvre artistique peut se réclamer d'une liberté de création totale et dépendre de la seule fantaisie de son auteur, il est vrai aussi que cette « autonomie » peut être relativisée par certains garde-fous que l'œuvre se donne elle-même, volontairement ou à son insu. Dans le premier cas, l'œuvre peut se rattacher à de grandes catégories génériques et faire sens par la façon dont elle parvient ou non à respecter le cadre qu'elle s'est imposé. Dans le deuxième cas, le «sens » fait corps avec les stratégies subtiles auxquelles recourent les auteurs pour camoufler leurs motivations profondes. Dans les deux cas, les choix paradigmatiques opérés par les auteurs, dans les matériaux ou dans leur combinatoire, supposent toujours des positionnements idéologiques insidieux ou des ralliements à des "vérités » établies. De sorte que l'œuvre la plus louable dans son manifeste peut s'avérer, après analyse, le discours le plus conservateur et le plus « réactionnaire » qui soit. On connaît la sévère diatribe de Roland Barthes à l'encontre de la littérature française, diverse dans ses intentions, mais « bourgeoise » de par son écriture :

C'est toujours la même écriture dix-huitiémiste, témoin des fastes bourgeois, qui reste la norme du français de bon aloi, ce langage bien clos, séparé de la société par toute l'épaisseur du mythe littéraire, sorte d'écriture sacrée reprise indifféremment par les écrivains les plus différents, à titre de loi austère ou de plaisir gourmand (Barthes 42).

Plus largement, la poétique avec Roman Jakobson a établi qu'il existe deux niveaux de compréhension des textes, l'un qui repose sur l'instrumentation intentionnelle de la langue (fonction conative) et l'autre plus implicite, ou fonction poétique, qui relève d'une certaine faculté du langage à se produire pour "son propre compte », à exister de par lui-même (Jakobson 218). De même, Jean Ziegler, reprenant Max Horkheimer, distingue deux dimensions antagonistes du texte qu'il nomme "raison subjective et raison objective ». Cette dernière étant le niveau où le

\footnotetext{
${ }^{1}$ Par exemple, au premier festival de B.D. de Saint Louis au Sénégal et à la 14e biennale du festival international de la BD d'Erlangen en Allemagne (1 ${ }^{\text {er }}$ prix «Max Und Moritz»»).
} 
texte se construit un sens qui échappe à toute intentionnalité esthétique ou thématique de l'écrivain et n'existe que par la portée dialectique à reconnaittre aux choix formels effectués. Il en résulte une portée de l'œuvre qui dépasse son propre auteur, qui peut exister en dehors de la volonté de ce dernier, voire contre sa volonté : «La raison subjective de l'auteur - ses préférences politiques, sa conduite concrète de sa vie, ses jugements de valeur esthétiques, politiques - ne joue qu'un rôle mineur. La raison objective parle à travers lui. Comme malgré lui » (Ziegler 1980 6566).

L'album Dipoula de Pahé et de Sti se range à première vue dans la catégorie des ouvres à ambition avouée, en même temps qu'il se situe, du point de vue de sa construction, dans une logique d'ambiguité. En effet, c'est une œuvre qui affirme d'emblée son engagement tout en se réclamant, de par le genre choisi (l'humour fantaisie), d'une totale liberté par rapport aux situations qui lui servent de référence.

Or, l'engagement ne peut se baser que sur la dénonciation d'une réalité incontestable pour l'auteur et ses lecteurs, sur la certitude que les faits restitués ou évoquées existent bien et sont d'une nature tellement scandaleuse qu'ils exigent une action contraire. Les faits accentués par le duo Pahé et Sti existent-ils bien dans le cadre de la réalité gabonaise et avec une telle gravité qu'il importe de les dénoncer? C'est ce dont les auteurs auraient dû s'assurer s'ils avaient voulu fournir une œuvre qui permette de reconnaitre la réalité derrière les brouillages de la caricature, de sorte qu'aucune équivoque ne plane sur les motivations réelles de la production. CEuvre en apparence engagée, Dipoula ne serait-elle pas un leurre qui cache ses véritables intentions derrière des stratégies habiles de séduction?

On pourrait nous arguer le fait qu'il s'agit d'une appropriation esthétique, l'essentiel étant dans la sensibilisation du public, en particulier du jeune public. À cela nous répondrions que l'effet à long terme de textes peut souvent être plus ravageur que leur impact positif immédiat. Il est à parier que les enfants occidentaux auxquels la bande dessinée a plu se sont faits par la même occasion une image du Gabon qu'ils garderont aussi longtemps qu'une image contraire ne leur sera pas proposée. Le danger est que le Gabon se réduise longtemps pour ces enfants à une représentation dévalorisante de l'Afrique, représentation à laquelle ils sont, à en croire le sociologue Pap Ndiaye, plus ou moins préparés par les mécanismes d'un long et profond processus de discrimination raciale :

S'il n'existe pas de "nature noire", il est possible d'observer une "condition noire" par laquelle on signale que des hommes et des femmes ont, nolens volens, en partage d'être considérés comme noirs, à un moment donné et dans une société donnée. C'est faire référence à des personnes qui ont été historiquement construites comme noires, par un lent processus de validation religieuse, scientifique, intellectuelle de la « race» noire, processus si enchâssé dans les sociétés modernes qu'il est resté à peu près en place, lors même que la racialisation a été délégitimée. (Ndiaye 2008)

Il importe donc de vérifier en toute objectivité, si le modèle dont cette image prétend être la caricature existe réellement, ou s'il est, au contraire, un produit du conditionnement de deux auteurs soumis non seulemement à ce processus de stigmatisation du Noir mais aussi à un système éditorial qui correspond mutatis mutandis à la description qu'Edward W. Saïd fait du système international des médias :

Ce système mondial qui produit et fait fonctionner conjointement la culture, l'économie et le pouvoir politique, ainsi que leurs dimensions militaires et démographiques, a une tendance institutionnalisée à produire des images transnationales démesurées, qui réorientent, au niveau international, la pensée et la réalité sociale. 
[...] Ce sont des images effrayantes au contenu mal défini : elles confèrent pouvoir moral et approbation à tous ceux qui les utilisent, dévalorisent moralement et criminalisent tous ceux qu'elles désignent (Saï 430) ${ }^{2}$.

Pour être tout à fait significative, cette vérification portera sur les aspects les plus importants de la dénonciation, ceux qui se veulent les plus proches de la «réalité », à savoir l'albinos et sa stigmatisation, l'orphelin et la politique d'adoption au Gabon, ainsi que les marques les plus prégnantes de la « couleur locale $»^{3}$.

\section{1) L'albinos et sa stigmatisation}

Pays complexe, à l'image de son découpage en 8 provinces et des 40 ethnies qui composent son paysage démographique, le Gabon n'est pas un espace qui se laisse facilement réduire à des stéréotypes. Dans ce pays, chaque individu, chaque groupe, chaque communauté, semble mesurer l'existence à l'aune de sa propre sensibilité, et la réalité peut être très changeante d'un endroit à un autre, d'un jour à un autre. Il faut avoir vécu cette complexité pour donner tout son sens à la boutade de Laurent Owondo selon laquelle Libreville, capitale et microcosme du pays tout entier, est une ville «qui affirme une chose et dit aussitôt le contraire» (Owondo 3). Il faut donc se montrer extrêmement circonspect si l'on veut mettre au jour les grandes lignes transversales qui permettent d'établir un caractère national ou une marque identitaire. C'est dire que les usages ou les pratiques, les habitudes ou les attitudes que Pahé et Sti dénoncent, s'ils peuvent exister, ne sont pas forcément symptomatiques ou caractéristiques du Gabon dans son ensemble. Ceci est vrai particulièrement dans le cas des Albinos.

Contrairement aux propos tenus à Armel Le Ny selon lesquels en «Tanzanie et au Burundi, les albinos sont victimes de chasses à l'homme qui peuvent aller jusqu'au meurtre » (Le Ny V acances), l'albinisme au Gabon n'est pas le lieu d'une traque ouverte, même si les formes qu'y adopte la répression peuvent être ponctuellement aussi criminelles et abjectes que dans les pays cités. D'ailleurs dans l'album Dipoula, Pahé prend bien garde d'évoquer de telles ignominies. Aucune planche ne fait allusion à une éventuelle traque ou à un meurtre qui serait exécuté pour des raisons quelconques, en particulier des nécessités rituelles ${ }^{4}$. Le mal vivre de Dipoula découle principalement de la stigmatisation qu'il endure à cause de sa «couleur»: c'est à cause de sa couleur qu'il est abandonné dès sa naissance par ses parents et c'est à cause de sa couleur que sa future amie Magali accueillera ses premières avances par un cinglant: «je veux pas que tu me

\footnotetext{
${ }^{2}$ Les propos d'Edward Saïd désignent précisément le «terrorisme » et le «fondamentalisme » religieux, mais pour les paraphraser dans le cas de l'album Dipoula, nous parlerons d'images dégradantes qui dévalorisent et stigmatisent tous ceux qu'elles désignent.

${ }^{3}$ Un tel travail d'authentification et de déconstruction/dé-formation (Saïd 376) est d'autant plus légitime qu'il permet d'apprécier le degré de conviction, sinon le processus de détournement d'un auteur réputé pour son franc parler et son incorruptibilité. Traduisant le sentiment général, Guy Rossatanga-Rignault présente l'œuvre locale de Pahé comme une contribution à l'éducation populaire. Lui, comme Lybeck, un autre caricaturiste, "s'inscrivent dans le mouvement» qui consiste à assurer «l'édification du peuple dans le cade d'un processus de 'changement de mentalité' [...], en rendant compte des 'choses du pays'. Ce faisant, ils mettent les Gabonais devant le miroir de leur réalité sociale quotidienne dont les traces de laideur sont si apparentes qu'on finit par ne plus le savoir, jusqu'au jour où l'un ou l'autre des caricaturistes la croque » (Rossatanga-Rignault 20).

${ }^{4}$ Malgré le caractère ignoble qu'ils peuvent prendre aujourd'hui (au même titre que l'excision ou certaines formes de mortifications), le meurtre rituel des albinos doit être compris comme une soumission atavique ou une déviation d'un système sacrificiel qui a longtemps représenté une des composantes majeures des sociétés africaines traditionnelles. Alfred Adler peut ainsi dire : «Dans la royauté moundang, comme dans toutes les royautés d'Afrique noire, [...] dans les cultes liés au pouvoir, une place essentielle est faite aux rites sacrificiels. Les obligations ou les prérogatives du souverain en cette matière sont même souvent un des traits les plus marquants sinon les plus spectaculaires des manifestations religieuses qu'on peut observer dans ce type de société » (Adler 149). La persistance des sacrifices d'albinos ne doit donc pas être envisagée comme un signe d'arriération, mais comme un phénomène de résistance culturelle qu'il importe de comprendre pour mieux le dépasser.
} 
suives comme ça sale albinos !!! » (Pahé/Sti 30). Face à ce rejet, Dipoula se mettra dans la tête de devenir noir à tous prix, se prêtant, bon gré mal gré, aux charlataneries de son ami Blazé qui prétend avoir un «livre de recettes mystiques qui peuvent [le] rendre noir» (Pahé/Sti 5) ou lui « donner un beau teint d'ébène » (Pahé/Sti 12). Cette obsession pourrait donner le sentiment que l'albinisme au Gabon se vit principalement comme une forme de "racisme », l'essentiel de la stigmatisation portant sur une certaine répugnance éprouvée à l'endroit d'un teint qui trancherait avec le noir généralisé. Mais ce serait présenter de façon extrêmement réductrice une situation qui résulte avant tout de la «modernisation » de sociétés traditionnelles fragilisées par la colonisation. Le mal du Gabon, petit pays par la superficie et la démographie, relève paradoxalement de ce qui a longtemps fait sa force, ses richesses minières présumées inépuisables. Durant le boom pétrolier (1970-1980), l'argent a coulé à flot, au point de devenir la valeur essentielle, l'attraction principale et l'aune en fonction de laquelle se mesurent la qualité, le mérite et la position sociale. Il infuse ainsi au cœur de sociétés à peine libérées du joug colonial une logique perverse, fondée sur les promesses d'ascension accélérées, les stratégies d'acquisition effrénée et les manœuvres les plus basses de confiscation. Dans un tel contexte, l'on se préoccupe peu de scrupule ou de partage, et mis à part les mécanismes résiduels d'entraide et de soutien, les politiques sociales se limitent à de simples déclarations d'intention. C'est dans ce cadre que se situe le rejet de l'albinos. Ce dernier se range dans la catégorie des faibles et des pauvres, entraves aux ambitions effrénées du parvenu ou repoussoirs à son élévation sans limites. Au même titre que l'enfant de la rue, l'orphelin ou tout autre handicapé, l'albinos représente la marginalité et la pauvreté honnies. Il est une source de dépenses inutiles et une incongruité manifeste qui peut contrarier le souci d'étalage. Le rejet des albinos est donc avant tout une tare de mauvaise gouvernance, il témoigne de l'incapacité des décideurs politiques à fournir aux albinos les conditions (sanitaires, psychologiques) indispensables à leur intégration. Il n'y pas au Gabon d'éviction ou de massacre systématique de l'albinos, certaines ethnies - comme l'ethnie fang a à laquelle appartient Pahé - le tenant à l'origine pour une «bénédiction» (Le Ny Vacances). La situation décrite en ouverture de l'album, d'un couple myénè qui se débarrasse de son enfant albinos par peur du «mauvais cail» (Pahé/Sti 3), sans être une hérésie, aurait été encore plus convaincante si au lieu d'arguer simplement du "mauvais œil », le couple avait justifié cette abjection par les difficultés financières monstres qui se profilaient ou par la pression inévitable du « qu'en dira-t-on ».

\section{2) L'orphelin et la politique d'adoption au Gabon}

Concernant l'orphelin que voudrait camper Dipoula, il représente au même titre que les autres « cas sociaux », une écharde à la volonté générale d'enrichissement et de promotion sociale. Il détonne ainsi dans un contexte où la course impitoyable pour l'argent inspire des réflexes de « chacun pour soi » ou de « donnant, donnant». Ce durcissement s'est à un tel point accentué que même les anciennes œuvres de bienfaisance qui bénéficiaient traditionnellement du soutien de l'Etat ont dû se réorganiser, sinon rompre avec leur fonctionnement ancien. Après avoir longtemps représenté le principal relais auprès des défavorisés, les œuvres religieuses, catholiques pour l'essentiel, se sont orientées - surtout à Libreville - dans la gestion d'établissements scolaires à caractère privé, soit entièrement autonomes, soit reconnus d'utilité publique. Le Ministère des Affaires Sociales peinant à remplir ses missions, le salut des orphelins est presque totalement entre les mains de certaines ONG, locales ou extérieures, qui se multiplient à cet effet. Les enfants sont soit mis en garde chez des parents proches, soit concentrés dans des centres d'accueil, pour des périodes plus ou moins longues, en fonction des difficultés rencontrées pour les placer ou pour les faire adopter. Ces enfants, dont l'âge peut aller du nourrisson à l'adolescent, bénéficient d'un encadrement urgentiste qui met l'accent sur la satisfaction des besoins élémentaires (alimentation, vêtements, etc.) et sur leur éducation scolaire. Au Gabon, où le principe de famille étendue reste encore très vivace, la «tare » d'être «orphelin » est forcément liée à des mécanismes de dérobades parentales couplés à des formes insidieuses de stigmatisations 
superstitueuses. C'est certainement la raison pour laquelle, le nombre le plus croissant d'orphelins est celui des orphelins du Sida. C'est en tous les cas le constat effectué par de nombreux responsables d'ONG, dont Mekontso Bekalé Marguerite, présidente de l'ONG « Lumière », en lutte contre le « rejet de l'orphelin ». S'en indignant sur le site allafrica.com, elle déclare : "Nous avons constaté sur le terrain que plusieurs personnes rejettent les orphelins du SIDA par ignorance, en disant que si leurs parents sont décédés du VIH, c'est que, eux aussi, sont nécessairement malades ». (Ntoutoume 2009). Il est donc de plus en plus fréquent de rencontrer des familles isolées d'orphelins, soumises à l'autorité des aînés et survivant grâce à des petits métiers. On le voit, l'image qu'offre l'album Dipoula d'un orphelinat tenu par des religieuses n'est pas véritablement représentative de la situation générale au Gabon. Cette caricature est non seulement injuste pour le travail fourni par les ONG, mais elle a aussi tendance à édulcorer les mécanismes complexes de la condition d'orphelin dans un pays encore soumis à de nombreuses traditions.

Ce décalage est tout aussi manifeste dans le traitement auquel le scénariste soumet le corollaire immédiat de l'orphelin, à savoir l'adoption. De fait, dans les scénarii de l'album, comme dans la réalité, l'adoption est le débouché incontournable de Dipoula et ses amis, l'accomplissement auquel doivent tendre leurs moindres vœux. Mais, sur ce plan aussi, la situation du Gabon est très particulière. En effet, les textes gabonais en matière d'adoption ne reconnaissent pas l'adoption internationale, sauf si l'adoptant est installé au Gabon. La hantise ou l'obsession de Dipoula à être adoptée est donc plausible, mais les potentiels adoptants qui se succèdent ne sont pas crédibles dans la mesure où ils ont tous l'air d'être des touristes de passage.

De même, l'album -, "vous avez combien de télés chez vous ? Chuis sûr qu'il a au moins 10.000 chaînes différentes en France!» (Pahé/Sti 42), "pas de grabuge pendant que nos amis français sont là (Pahé/Sti 43) «j’vais voir la France les gars!» (Pahé/Sti 46) pourrait faire croire que dans la réalité la majorité des adoptants sont exclusivement des « Français » expatriés, alors que le processus d'adoption officiel concerne aussi - et même surtout - les ressortissants gabonais. Cette disposition est d'autant plus importante que, au regard du fonctionnement traditionnel de la famille gabonaise, il est impossible pour un enfant de se retrouver sans parent consanguin, connu ou identifiable. Sa naissance le rattache d'emblée à un groupe de personnes extrêmement large dont le resserrement interdit toute possibilité d'exclusion volontaire. C'est la fameuse famille élargie dont les sociologues ont souvent fait état : «Au Gabon, la famille étendue représente l'un des éléments fondamentaux des rapports sociaux de groupe. Par ce caractère, elle est un processus d'entrée en communauté, un centre de refuge et de sécurité au sein duquel l'individu dispose de plusieurs relations-supports d'ordre socio-psychologique, procurant équilibre et protection» (Moussavou 40). Les enfants, recueillis par les centres ne sont donc pas des orphelins au sens strict, puisque les mécanismes de préservation du tissu familial doivent leur assurer une tutelle de substitution immédiatement après la mort de l'un ou des deux parents.

La situation d'ostracisme à laquelle sont confrontés les orphelins est l'expression de ces stratégies de dérobades ou de spoliations superstitieuses que nous avions déjà relevées. Mais quelquefois, la pression des centres ou le simple remord peut amener les "parents » à se raviser au point de redemander la garde de «leur enfant». L'image d'un orphelinat tourné exclusivement vers l'adoption internationale et occidentale comme unique débouché est donc elle aussi une entrave à la vérité culturelle et sociale. Tout comme est contraire aux démarches d'adoption officielle, toujours confidentielles et extrêmement procédurières, le processus d'adoption chez Dipoula, qui consiste en une vaste revue d'effectif par laquelle une ruée d'adoptants potentiels - « dépêchezvous les gars, le car des parents adoptifs français arrive » - sont présentés à un groupe d'enfants enrégimentés, contraints de « se mettre sur leur 31 » et encouragés à jouer de leur séduction pour 
se faire adopter : "vous allez vous mettre en petits groupes et allez vers les parents français afin de leur poser vos questions et de répondre aux leurs...»! (Pahé/Sti 41)

\section{3) Couleur locale, étiquettes, poncifs et interférences}

Si Pahé et Sti peuvent revendiquer les libertés de la bande dessinée pour justifier les accommodements pris avec la réalité, il n'en reste pas moins qu'ils manifestent un certain souci de respecter cette réalité. Sti a besoin de séjourner à Libreville pour s'imprégner de la couleur locale et garantir une certaine vraisemblance à ses scénarii. Mais l'immersion n'est pas suffisante puisque, comme Pahé le confesse à Nicolas Vadeau, même si « les histoires sont donc de Sti », il doit souvent rattraper son scénariste, en intervenant beaucoup sur la vraisemblance de l'histoire (Vadeau Rencontre).

Pahé reconnaît de la sorte qu'un travail d'authentification et de contrôle s'imposait pour concilier un minimum d'honnêteté et de vraisemblance, mais la contrainte des délais ou le côté accrocheur de certains scénarii l'ont conduit à tolérer des aspects qui, pour être moins grossiers, n'en restent pas moins discutables. Ces détails révèlent chez Sti une tendance à substituer à la situation réelle, dans sa complexité, la déformation caricaturale dont il est fortement imprégné. Plus loin même, cette vision a priori pourra conduire le scénariste à imposer à la réalité gabonaise, qu'il veut restituer, le substrat de sa culture occidentale.

Le premier trait concerne à nos yeux l'image d'Epinal d'un orphelinat tenu par les sœurs. Bien que les religieuses se soient progressivement départies de la gestion des institutions de bienfaisance, l'image reste ancrée d'une Afrique livrée à la bonne volonté et à l'altruisme de ces «servantes » du Christ. Le trait est d'autant plus accentué que les religieuses en question sont toutes des «Blanches», comme s'il fallait reproduire le schéma des premières missions évangélisatrices au Gabon. Or, au Gabon, le clergé s'est depuis longtemps métissé au point que les religieuses sont en majorité africaines, et les dernières sœurs «blanches » qui demeurent sont surtout de nationalités... sud américaines.

Un autre trait de cet arrimage à la mythologie coloniale est trahi par la présence incongrue d'un instituteur «blanc», un certain «Mr Toubab» aux allures de vieux baroudeur grincheux. Malheureusement pour la trouvaille, un instituteur «blanc» dans une école publique gabonaise cela ne s'est plus vu depuis... Mathusalem. Les derniers instituteurs français au Gabon enseignent dans des écoles dites "conventionnées", subventionnées à la fois par l'Etat français et par le gouvernement gabonais. De telles écoles pratiquent des tarifications qui les rendent largement inaccessibles à des revenus gabonais moyens, à plus forte raison ceux d'une armée d'orphelins. Pour le reste des écoles, le remplacement des instituteurs blancs par les premiers auxiliaires commence officiellement par « un arrêté du Gouverneur Général du 30 novembre 1926 [qui institue] un cadre local des moniteurs indigènes dans chacune des colonies de l'A.E.F. destiné à seconder le personnel européen de l'enseignement» (Essono-Edzang 342). Ce processus s'est poursuivi depuis lors, au point que les effectifs des enseignants du premier degré sont constitués à près de 100\% par des instituteurs gabonais. Camper dans le Libreville d'aujourd'hui un instituteur «blanc » au service d'une école de « quartier », c'est se rattacher à l'époque désuète où l'Europe s'était donnée pour mission de « civiliser » l'Afrique.

Une autre forme de fixation à l'imaginaire colonial est fournie par le fait que les infirmières de la Croix Rouge, à l'instar des religieuses ou de l'instituteur, sont blanches, en particulier la superbe «Adriana Karembol » dont les courbes plantureuses suscitent bien des pensées lascives chez nos petits pensionnaires. Or, non seulement les volontaires de la Croix Rouge au Gabon ne portent plus d'uniformes, mais elles ne sont plus «blanches», pas plus qu'elles ne sont forcément 
infirmières, depuis que la Croix Rouge fonctionne selon le mode de l'antenne locale et non de la représentation internationale.

On le voit, le regard que porte Sti sur le Gabon est déformé par des clichés qui le ramènent à la colonisation. Cette impression est encore plus vive lorsque le scénariste s'empare de poncifs aussi éculés que ceux du singe ou du gorille tropicaux. Le gorille à proximité des cases est digne des meilleurs délires hollywoodiens, alors que l'anecdote d'un singe que l'on élève et engraisse comme un cochon ne peut faire rire aucun enfant gabonais. Parlant justement du cochon, la figure du cochon Gruik, mascotte du petit groupe d'orphelins, viole toutes les références gabonaises en matière de bestiaire. Le cochon, sans être un animal tabou au Gabon, n'en reste pas moins le symbole par excellence de la bêtise et de la gourmandise. Il ne viendrait jamais à l'esprit d'un enfant gabonais, même un orphelin ou un enfant de la rue, d'en faire son compagnon de jeu. Dès lors, l'amitié établie entre Dipoula et Gruik peut être révélatrice d'un rapprochement particulièrement désobligeant entre le cochon et l'Africain... ${ }^{5}$

Passant sur d'autres lieux communs comme l'habillement «typique » des femmes (camisole et pagne) ou l'inévitable étonnement suscité par la diglossie - «en plus il parle déjà français » (Pahé/Sti 44) -, il convient de relever dans le texte l'intrusion de références qui sont propres à la culture du scénariste. Ce sont, par exemple, les commerces qui sont tous fermés le dimanche ou encore le dialogue final entre la mère supérieure et le christ en croix, digne d'un « entretien » entre Don Camillo et Jésus. Il faut dès lors admettre que derrière « les réalités du pays » restituées par Sti, se profile le substrat d'une culture occidentale qui, non seulement édulcore la société gabonaise, mais va jusqu'à la doter de ses propres repères. Comment dès lors ne pas penser que derrière le Dipoula de Sti/Pahé, variante africaine du chenapan, se profile l'archétype occidental du galopin frondeur et paresseux, nous avons nommé Titeuf?

\section{3) Dipoula : un Titeuf mal noirci ?}

À en croire de nombreux chroniqueurs, il voudrait mieux ne pas affirmer en face de Pahé que Dipoula est un Titeuf africain. Ainsi Armel Le Ny déclare : «Ne dites pas de Dipoula qu'il est le Titeuf noir. D'abord parce que cela mettrait en rogne Pahé, son papa, et surtout parce que c'est faux: le petit orphelin est blanc, et même plus blanc que blanc: albinos » (Le Ny V acances). Et c'est presque mot pour mot le même sentiment chez JeuneAfrique.com : "Impossible de ne pas penser au héros des cours de récrés françaises, Titeuf. Alors Dipoula, un Titeuf africain ? Son papa se fâche : il a le même âge, mais ça n'a rien à voir. C'est un orphelin albinos ! Je pense plutôt que Titeuf c'est le Dipoula français! » (Nicolas Terreur)

Comment expliquer une telle phobie chez Pahé ? Serait-elle une simple réaction d'orgueil ? À moins que ce comportement ne voile un remords intérieur, comme le sentiment de s'être prêté à un maquillage volontaire ? N'avouera-t-il pas à Nicolas Vadeau que devant «le syndrome de la page blanche » (Vadeau Rencontre), il a dû laisser toute latitude à Sti, n'intervenant que pour corriger quelques incongruités trop grossières. Comment ne pas penser que le scénariste lillois, au moment de camper un personnage d'enfant pétulant et frondeur, ait songé au personnage blanc

\footnotetext{
${ }^{5}$ Une fois n'est pas coutume, notre effort de décryptage et de contextualisation peut aussi aider à éviter des méprises dans l'interprétation de Dipoula. La présence de Cissoko, un orphelin ouest-africain vêtu d'une gandoura et d'une chéchia, peut donner l'impression que Pahé salue l'adhésion de la famille présidentielle gabonaise à l'islam, même si, vu du Gabon, le statut officiel de pays musulman qui en découle ( $0,2 \%$ de Gabonais de souche) ne représente qu'une stratégie politique ou un « coup » diplomatique. Mais pour qui connaît bien la situation démographique du Gabon, il est plus plausible que Pahé ait voulu restituer le partage de la population en une majorité de Gabonais de souche, renforcée par une minorité de «Popos» ou Africains de l'Ouest, lesquels ne sont pas tous musulmans, le boubou long (gandoura) et la chéchia n'étant pas systématiquement le signe d'une culture islamique.
} 
qui en est l'archétype par excellence ? Il suffit de vérifier combien Dipoula doit à Titeuf, autant sur le plan physique que sur celui de la logique actionnelle ou des référents mythologiques, pour comprendre à quel point l'orphelin albinos n'est qu'un travestissement du petit « morveux » à la crête blonde.

\section{1) Dipoula et son ombre}

N'en déplaise à Pahé, il est difficile de découvrir Dipoula sans penser de suite à Titeuf. Les deux personnages ont à peu près la même taille, Pahé ayant retiré quelques centimètres à son personnage d'origine pour lui donner les allures « d'un enfant de la rue ». Ils ont aussi en commun une tête démesurée par rapport au reste du corps. À la chevelure en houppe ou en panache de Titeuf correspond chez Dipoula une curieuse protubérance qui pourrait évoquer une coiffure de cheveux très clairs. Si le nez très important chez Dipoula pourrait permettre d'établir une différence très nette, il est tellement développé et collé aux mâchoires qu'il paraît être la même énorme bouche que celle de Titeuf. Les deux visages donnant ainsi l'impression d'une poire s'évasant exagérément sur la base. Bien entendu, Pahé pourrait brandir la défense qu'il a conçu son personnage bien avant qu'il n'ait connu Titeuf. Mais un dessin peut encore évoluer, en fonction des influences et des intentions. Les aménagements effectués sur Dipoula vont certainement plus loin que la seule amputation de quelques centimètres. Il est possible que Pahé ait très finement accentué la ressemblance entre Dipoula et Titeuf, en évitant de la rendre trop aveuglante... C'est ce qui expliquerait probablement sa nervosité chaque fois qu'il est question de cette ressemblance...

\section{2) Des motifs et des thèmes en partage}

Invité à trouver en urgence des scénarii qui mettent en valeur un garnement pétulant mais sympathique, Sti ne peut agir autrement qu'en s'appuyant sur ses références personnelles. Malgré sa bonne volonté et le désir de faire couleur locale, il ne pouvait que s'inspirer des motifs ou des thèmes qui articulent le monde de Titeuf, modèle obvie du chenapan au grand cœur. Les plus marquants de ces motifs sont le rejet de l'école, l'incompréhension du monde des adultes, la capacité de déformer ce monde au moyen du prisme enfantin, l'univers secret des enfants, le cercle intime de leurs amitiés, etc.

Concernant l'école, à l'instar de Titeuf, Dipoula sera un élève médiocre, multipliant les mauvaises notes et assimilant le moindre effort intellectuel à une corvée insipide. Comme son pendant français, on le voit brandir des copies affichant le détestable 0/10 attribué par un Mr Toubab qui, à l'instar de l'institutrice hommasse de Titeuf, n'inspire à son élève qu'une envie d'apprendre soporifique. Anti-héros par excellence, il sera adepte du médiocre, mauvais sportif, mauvais ami, menteur, gaffeur, multipliant les pets et les rots, et traînant ses chaussures boueuses sur les draps blancs des mensonges adultes. Seuls l'amitié de ses "potes» et les stimuli de l'amour sauront amener de l'éclat dans sa vie. Amour pour Magali, pendant africaine de la Nadia chère à Titeuf, amour impossible pour Adriana Karembol, une infirmière de la Croix Rouge aux charmes de pinup dont les homologues hantent les « expériences » de Titeuf.

Autrement, de nombreux autres motifs sont reconnaissables pour celui qui a bien lu son Titeuf. Ce sont, entre autres, la punition qui consiste à laver les toilettes «cradingues » de l'école, le gag qui consiste à faire prendre des crottes (de singe) pour des dragées, le cochon Gruik qui remplace Brutus le gros dogue de Manu, les courses haletantes pour se mettre à l'abri d'une bastonnade, les courriers truffés de fautes adressés à différentes «victimes », etc. Autant de motifs chers à Titeuf, qui prennent avec Dipoula une certaine coloration africaine, sans qu'il soit possible de dissiper une impression de déjà vu... 


\section{3) Une logique similaire du récit}

La concordance établie entre Titeuf et Dipoula au niveau des motifs se concrétise dans le recours à un style narratif globalement classique. Les deux albums fonctionnent comme une succession de planches dont chacune raconte sa propre histoire. Quelquefois, l'histoire peut s'étendre sur deux planches ou sur bien plus, comme chez Sti et Pahé, dont les huit 8 dernières planches sont consacrées à l'adoption ratée de Dipoula. Mais le schéma type est celui d'une planche (de 5 à 8 cadres [en moyenne] chez Pahé, jusqu'à 12 cadres chez Zep), laquelle présente une histoire linéaire allant d'une scène initiale d'exposition à une chute en forme de coup de théâtre. Chaque récit est introduit par un titre, avec la particularité pour Dipoula d'amorcer quelquefois son récit par un récitatif à tonalité autobiographique.

Mais l'essentiel reste la gestion de la satire, avec les techniques courantes d'une bande dessinée caricaturale, à savoir le changement de perspective ou de plan entre l'avant-dernière page et la dernière, ou encore l'illusion de l'aparté et la connivence avec le lecteur chère au genre théâtral, etc. Autrement, les deux récits se caractérisent par une chute toujours déceptive, sauf pour la dernière planche de Dipoula qui semble une tentative presque maladroite de rattraper la tonalité pessimiste d'ensemble. Au contraire des personnages fondamentalement sympathiques (Boule et Bill) ou même des gaffeurs malgré eux (Gaston Lagaffe), Dipoula et Titeuf se caractérisent par une logique d'enfant terrible susceptible de faire chaque chose «à l'envers » (Calame-Griaule 29), au point que ce qui peut paraittre sur l'instant une victoire sur les autres (comme de regarder Adriana Karembol à la télé à l'insu des sœurs) peut s'avérer à la longue une défaite par rapport à eux-mêmes.

\section{En guise de conclusion}

Nous aurons donc vérifié tout le long de notre parcours combien Dipoula emprunte à Titeuf aussi bien dans la représentation des « réalités gabonaises » que dans la structuration de l'album. La question est dès lors de savoir si les auteurs (et producteurs) étaient conscients de cette « ressemblance » et, dans l'affirmative, d'expliquer leur silence ou leur crispation à ce propos. À notre avis, les trois collaborateurs ne pouvaient qu'avoir - ou prendre - conscience des liens qui unissent Titeuf à Dipoula, Titeuf représentant une sorte d'archétype pour le "caractère » type de garnement adoré. La dissimulation ou l'agacement affiché par Pahé chaque fois qu'il est question de cette ressemblance viendrait ainsi traduire la crainte de voir mettre à nu les stratégies diverses que sous-tend le camouflage plus ou moins ouvert du célèbre garnement. De sorte que l'on pourrait interpréter la démarche de chaque partenaire en fonction du profit qu'il pourrait tirer de l'entreprise. Dans ce cadre, Sti nous semble le moins répréhensible. Pressé d'accoucher de scénarii vraisemblables et accrocheurs, il doit se contenter d'une courte semaine d'observation à Libreville et s'en remettre pour le reste à ses propres référents culturels. Qu'il ait trop penché vers Titeuf, au point d'en avoir représenté un ersatz, c'était une dérive qu'il incombait au dessinateur ou au producteur de redresser.

Justement, Pahé et son producteur nous semblent vivre la relation de décalage sémantique que dépeint Babette Cole dans l'album «Le problème avec ma mère » (Cole 1983). Elle y construit une forme de mise en abyme où un "garçon raconte avec un sérieux parfait les difficultés qu'il a avec sa mère car il ne se rend pas compte qu'elle est une sorcière » (Beau 58). De même, Pahé affirme avec une parfaite innocence des choses qui prennent une autre dimension une fois appliquées au langage de la production. La nécessité d' "internationaliser» Dipoula peut facilement se traduire en l'intérêt d'adapter le personnage porteur de l'albinos aux goûts et aux habitudes du jeune public occidental. Tant mieux si la plume de Sti a imprudemment dérivé ou si 
le crayon de Pahé s'est prêté à la subversion. Il semble bien que la donnée économique ait fondamentalement primé au point de réduire en artifices les bonnes intentions affirmées. Sous le prétexte de la cause humanitaire et de l'engagement antidiscriminatoire, c'est bien le bénéfice du producteur et le succès des auteurs qui étaient visés. C'est d'ailleurs ce que reconnaît Pahé, même s'il se cache derrière l'adhésion massive de son jeune public à la cause des albinos. Mais pour quel impact et à quel degré ? Ne faut-il pas plutôt craindre que les poncifs accumulés (singe, cochon, religieuses blanches, etc.) viennent renforcer une vision péjorative de l'Afrique ? Pahé en est certainement conscient. Mais il se prête au jeu, parce que la situation l'exige, parce que comme l'affirme Sébastien Langevin, "très peu d'auteurs de bande dessinée vivent de leur art en Afrique » et parce que "l'édition est le maillon faible, voir manquant, de la chaîne qui devrait permettre aux auteurs de B.D. de trouver leur public » (Langevin 30-31).

D'où le recours aux facilités et aux opportunités que lui autorise l'expatriation en Occident, au cœur de la métropole francophone. Une fois son talent reconnu, il lui est possible de bénéficier de conditions de travail et de production dignes des meilleurs spécialistes français. Mais à quel prix ? En se pliant aux règles pragmatiques de la production à grand tirage. Mais le dommage peut être plus grave et faire plus de victimes collatérales qu'il ne croit. Détenteur du pouvoir financier, magicien capable de transformer le «vilain petit canard» en cygne majestueux, le producteur peut amener l'auteur africain à conforter subtilement une perception valorisante de l'Europe. En l'amenant, par exemple, à effacer les traces de la tension originelle qui persistent depuis l'esclavage et la colonisation. Partant d'une médiatisation caricaturale des exactions (réelles) perpétrées contre les albinos en Afrique, Sti et Pahé, sous le couvert du rire, contribuent à faire porter à la seule Afrique le poids de telles ignominies, en les assimilant à des formes de rejet ou à des superstitions ataviques, sinon barbares. Notre propos aura consisté à complexifier la situation, en établissant que les crimes contre les albinos ne sont d'abord un héritage malsain de l'histoire, le maintien ou la survivance de pratiques anachroniques dans le contexte de sociétés en crise, fragilisées par la pénétration coloniale. Elles constituent ainsi des marques de mauvaise gouvernance dans la mesure où les élites politiques ne parviennent pas à poser les bases d'un état harmonieux, en phase avec son passé et avec les nouvelles exigences de la planétarisation. Or, quelles sont ces élites politiques? Ne relèvent-elles pas d'un système de proto-nation, cette "sociabilité rudimentaire, limitée dans sa construction, asservie aux seuls besoins de ceux qui l'organisent de l'extérieur. [...] avant tout une création de l'impérialisme » (Ziegler 1980 7-8).

Sous cet angle, le beau projet pédagogique du dessinateur ne s'avère en dernière instance que l'habile manœuvre d'un "désorienteur»! (Ziegler 1980 8) ${ }^{6}$ On est loin, bien loin de la vraie francophonie, cette "francophonie de demain, celle qui ne sera fondée ni sur la charité ni sur la mendicité, mais sur le dialogue libre et fraternel entre deux civilisations, entre la langue française et les langues négro-africaines » (Gassama 13).

\footnotetext{
${ }^{6}$ Ce néologisme forgé par Franz Fanon (Les damnés de la terre) et repris par Jean Ziegler (Ziegler 8), désigne dans la situation africaine d'aujourd'hui, une catégorie de personnes, intermédiaires entre le pouvoir dictatorial et le peuple, dont le rôle est de proposer par l'exemple de leur " réussite », par leur adhésion à un mode conventionnel de pensée (ou faussement progressiste), un modèle d'ascension sociale qui devrait maintenir insidieusement la majorité sous le joug de l'aliénation.
} 


\section{Bibliographie}

\section{A. Corpus de base}

PAHE \& STI, Dipoula (Mbolo). Paris : Paquet, 2008.

\section{B. Textes de référence}

Adler, Alfred. Le pouvoir et l'interdit. Royauté et religion en Afrique noire. Paris : Albin Michel, 2000. Barthes, Roland. Le degré zéro de l'écriture. Paris : Seuil, 1972.

Beau, Nadine. "Les différentes composantes d'un album», Notre Librairie, Guide pratique de l'illustrateur, s.n./hors série (janvier-mars 2003) : 53-58.

Calame-Griaule, Geneviève. « Propos recueillis par M.C. JACQUEY. » Notre Librairie 4243 (juillet-septembre 1975) : 29-30.

Cole, Babette. Le problème avec ma mère. Paris : Seuil, 1983.

Couturier, Jean-Louis. «À propos des styles graphiques dans la B.D. d'Afrique. » Notre Librairie 145 (2001) : 14-19.

Essono-Edzang, Aristide. Etude d'une société : Les auxiliaires indigènes de l'autorité coloniale en Afrique équatoriale française (A.E.F.). Bordeaux III : Thèse de doctorat, 1993.

Langevin, Sébastien. «Edition et diffusion de la B.D. d'Afrique : vaincre la marginalité. » Notre Librairie 145 (2001) : 30-33.

Gassama, Makhily. La Langue d'Ahmadou Kourouma ou le français sous le soleil d'Afrique, Paris : ACCTKarthala, 1995.

Jakobson, Roman. Essais de linguistique générale. Paris : Minuit, 1963.

Moussavou, Georges. «Formes et modèles de socialisation politique des étudiants gabonais en France et au Gabon, Contribution à une sociologie de l'identité. » Le Gabon: Approche pluridisciplinaire. Paris : L'Harmattan, 2006, 37-56.

Ndiaye, Pap. La Condition noire. Essai sur une minorité française. Paris : Calmann-Lévy, 2008.

Owondo, Laurent. «La vitrine du bord de mer. » Revue Noire 5 (1992) : 3.

Rossatanga-Rignault, Guy. «Lybek et Pahé : avant-garde intellectuelle du Gabon?» Créations littéraires et artistiques au Gabon. Les savoirs à l'cuvre. Libreville: Raponda Walker, 2009.

Saïd, Edward. Culture et impérialisme. Paris : Fayard/Le Monde diplomatique, 2000.

Ziegler, Jean. Main basse sur l'Afrique. La recolonisation. Paris : Seuil, 1980.

Ziegler, Jean. "La puissance du verbe. » La langue d'Abmadou Kourouma on le français sous le soleil d'Afrique. Paris : ACCT-Karthala, 1995, 7-9.

\section{Webographie}

\section{A/ Entretiens avec Pahé}

Bangré, Habib, «Dipoula, le super héros albinos. » 20 février 2009. 10 janvier 2010. <http://www.afrik.com/article16197.html>

Le NY, Armel, «En vacances avec Dipoula le petit albinos.» 10 juillet 2009. janvier 2010 $<$ http://blog.charentelibre.com/journal/index.php?post/2009/07/04/3380-en-vacances-

avec-dipoula-le-petit-albinos $>$

Michel, Nicolas, «Dipoula, la terreur de l'orphelinat.» 20 janvier 2009, 10 janvier 2010. <http://www.jeuneafrique.com/Article/ARTJAJA2506_p074-076.xml3/bande-dessineepahedipoula-terreur-de-l-orphelinat.html>

Vadeau, Nicolas, "Rencontre avec Pahé - Dessinateur de Dipoula. » 27 décembre 2008, 10 janvier 2010. <http://www.bdencre.com/interview-flash.html?2008/12/27/1945rencontre-avec-pahe-dessinateur-de-dipoula $>$

\section{B / Situation des orphelins du Sida au Gabon}


Ntoutoume, Douglas Anselme. AllAfrica.com. décembre 2009, 10 janvier 2010 $<$ http:/ / fr.allafrica.com/stories/200912011129.html> 